Rev Inv Vet Perú 2015; 26(1): 43-48

http://dx.doi.org/10.15381/rivep.v26i1.10911

\title{
Características Bioquímicas del Plasma Seminal Fresco y Congelado/ Descongelado de Alpaca (Vicugna pacos)
}

\author{
Biochemical Characteristics of Fresh and Freeze/Thawed Seminal Plasma of Al- \\ PACA (VICUGNa PACOS)
}
Hugo Díaz V. ${ }^{2}$, Juan Espinoza B. ${ }^{2}$, Wilfredo Huanca L. ${ }^{3}$, Bernardo Lopez-Torres ${ }^{2}$, José Rodríguez G. ${ }^{1,4}$

\section{Resumen}

\begin{abstract}
El objetivo del estudio fue determinar y comparar las características bioquímicas del plasma seminal de alpacas en fresco y descongelado. Se recolectó semen, mediante electroeyaculación, de cuatro alpacas adultas, una vez por semana por cuatro semanas. El semen se centrifugó y el plasma seminal fue separado. Una parte se analizó en fresco y la otra parte se almacenó en nitrógeno líquido por un mes. Se le hizo el análisis bioquímico a ambos juegos de muestras. Se determinaron los niveles de glucosa, colesterol total, colesterol-HDL, triglicéridos, proteínas totales, albúmina, calcio, fosfatasa alcalina, ALT y $\gamma$-GT. Solo los valores de triglicéridos descendieron significativamente por el proceso de congelación/descongelación ( $p<0.05$ ), siendo los valores de $44.12 \pm 7.38$ y $27.31 \pm 4.65$ $\mathrm{mg} / \mathrm{dl}$ en fresco y descongelado, respectivamente.
\end{abstract}

Palabras clave: bioquímica de plasma seminal; congelación/descongelación; alpacas

\section{Abstract}

The aim of this study was to determine and compare the biochemical characteristics of fresh and thawed seminal plasma of alpacas. Semen was collected by electroejaculation from four adult males, once a week per four weeks. Semen was centrifuged and the seminal plasma was separated. One part was analysed fresh and other stored for one month on liquid nitrogen and then thawed and analysed. Levels of glucose, total cholesterol, HDL-cholesterol, triglycerides, total protein, albumin, calcium, alkaline phosphatase, ALT and $\gamma$-GT were determined. Only the triglycerides significantly decreased due to the process of freeze/thawed, where the values were 44.12 \pm 7.38 and $27.31 \pm 4.65 \mathrm{mg} / \mathrm{dl}$ in fresh and thawed respectively.

Key words: biochemistry of seminal plasma; freeze/thawed; alpacas

\footnotetext{
${ }^{1}$ Estación Experimental del Centro de Investigación IVITA - El Mantaro, Huancayo, Perú

${ }^{2}$ Laboratorio de Farmacología y Toxicología, ${ }^{3}$ Laboratorio de Reproducción Animal, Facultad de Medicina Veterinaria, Universidad Nacional Mayor de San Marcos, Lima, Perú

${ }^{6}$ E-mail: joserodriguezmv@gmail.com
}

Recibido: 10 de junio de 2014

Aceptado para publicación: 30 de octubre de 2014 


\section{INTRODUCCIÓN}

El conocimiento de la biología reproductiva de los camélidos sudamericanos sigue siendo de gran interés, especialmente en lo referido a la fisiología reproductiva del macho. La inseminación artificial es una de las tecnologías que ha contribuido al progreso genético en diversas especies de animales de producción, especialmente en el bovino; sin embargo, la información sobre la colección, características, evaluación y conservación del semen en la alpaca es aún un campo abierto a la investigación, debido a que se van adecuando metodologías que sean reproducibles, especialmente en lo referente a la colección de semen (Ferré y Werkmeister, 1996). Asimismo, se va obteniendo más información sobre la composición bioquímica del plasma seminal, viscosidad del semen y uso de dilutores en la alpaca (Bustinza, 2001).

El plasma seminal consiste en una compleja mezcla de secreciones que se originan principalmente en el epidídimo y las glándulas sexuales accesorias del macho (TöpferPetersen et al., 2005). Este cumple un rol de protección de los espermatozoides dentro del tracto reproductivo de la hembra (Troedsson et al., 2005), donde, por ejemplo, las proteínas presentes en el plasma seminal protegen selectivamente a los espermatozoides vivos para no ser fagocitados por los polimorfonucleares neutrófilos presentes en el útero $y$, asimismo, juegan un rol importante en el transporte y eliminación de espermatozoides muertos (Loomis, 2006).

El plasma seminal participa en la maduración final del espermatozoide a través de cambios hormonales, enzimáticos y modificación de la superficie de membrana espermática. Además, sirve como vehículo para los espermatozoides (Muiño-Blanco et al., 2008). Parte de la amplia variación de la calidad espermática del semen refrigerado que se observa entre machos de una misma especie puede atribuirse a diferencias en la composición de su plasma seminal (MuiñoBlanco et al., 2008).

El semen de la alpaca es altamente viscoso, lo que hace difícil su manejo en el laboratorio. Asimismo, la viscosidad del semen influye para que no ocurra la motilidad masal y afecte la motilidad individual, la cual es de tipo oscilatoria y muy lenta (Sumar, 1997). La dificultad en la colección del semen es atribuida, entre otras causas, a las características peculiares de monta (posición de cúbito ventral), que dura entre 20 y 30 minutos. Por otro lado, se debe hacer notar que las características físicas del semen de la alpaca son muy semejantes a las de la llama (Garnica y Achata, 1998).

En el metabolismo del semen, el proceso respiratorio sigue los mismos pasos del ciclo de Krebs en presencia de oxígeno, utilizando para ello azúcares (glucosa, fructosa y manosa en el toro). En el caso del semen humano, también se observa la utilización de glucógeno y maltosa (De Alba, 1985). Otros componentes bioquímicos, como el colesterol, tienen como función la orientación reguladora y el flujo de la membrana lipídica (Cross, 1998). Estudios sobre lípidos demostraron que el incremento de lipoproteínas de muy baja densidad (VLDL) y los triglicéridos estaban correlacionados con la disminución de las características de motilidad del espermatozoide (Ergün et al., 2007).

Con base a esto, el objetivo del presente estudio fue determinar y comparar las características bioquímicas del plasma seminal de alpacas en el semen fresco y descongelado.

\section{MATERIALeS y MéTOdos}

El presente estudio se llevó a cabo en la Facultad de Medicina Veterinaria (FMV) de la Universidad Nacional Mayor de San Marcos, Lima, Perú. Se utilizaron cuatro alpacas machos de 3 a 5 años de edad, que fueron criadas bajo las mismas condiciones de ma- 
Cuadro 1. Comparación bioquímica del plasma seminal fresco y descongelado por alpaca

\begin{tabular}{|c|c|c|c|c|c|c|c|c|}
\hline & \multicolumn{2}{|c|}{ Macho 1} & \multicolumn{2}{|c|}{ Macho 2} & \multicolumn{2}{|c|}{ Macho 3} & \multicolumn{2}{|c|}{ Macho 4} \\
\hline & $\mathrm{F}^{1}$ & $\mathrm{C} / \mathrm{DC}$ & $\mathrm{F}$ & $\mathrm{C} / \mathrm{DC}$ & $\mathrm{F}$ & $\mathrm{C} / \mathrm{DC}$ & $\mathrm{F}$ & $\mathrm{C} / \mathrm{DC}$ \\
\hline $\begin{array}{l}\text { Glucosa } \\
\text { (mg/dL) }\end{array}$ & 9.07 & 7.37 & 7.49 & 8.67 & 8.43 & 9.45 & 7.88 & 8.64 \\
\hline $\begin{array}{l}\text { Colesterol } \\
(\mathrm{mg} / \mathrm{dL})\end{array}$ & 81.13 & 81.39 & 78.97 & 80.15 & 77.67 & 84.8 & 81.35 & 80.64 \\
\hline $\begin{array}{l}\text { Triglicéridos } \\
(\mathrm{mg} / \mathrm{dL})\end{array}$ & $47.33^{\mathrm{a}}$ & $24.84^{\mathrm{b}}$ & $49.57^{\mathrm{a}}$ & $27.83^{b}$ & 40.61 & 29.41 & 38.97 & 27.17 \\
\hline $\begin{array}{l}\text { HDL colesterol } \\
(\mathrm{mg} / \mathrm{dL})\end{array}$ & 4.56 & 4.64 & 4.91 & 4.96 & 4.79 & 5.17 & 4.67 & 5.09 \\
\hline $\begin{array}{l}\text { Proteína total } \\
(\mathrm{g} / \mathrm{dL})\end{array}$ & 2.3 & 2.16 & 2.5 & 2.41 & 2.27 & 2.16 & 2.37 & 2.42 \\
\hline $\begin{array}{l}\text { Albúmina } \\
(\mathrm{g} / \mathrm{dL})\end{array}$ & 1.09 & 0.74 & 1.13 & 0.85 & 0.82 & 0.81 & 0.86 & 0.84 \\
\hline $\begin{array}{l}\text { Calcio } \\
(\mathrm{mg} / \mathrm{dL})\end{array}$ & 11.66 & 11.04 & 11.27 & 11.71 & 11.83 & 11.1 & 12.31 & 11.91 \\
\hline $\begin{array}{l}\text { ALT } \\
\text { (U/L) }\end{array}$ & 12.22 & 6.05 & 20.31 & 16.8 & 18.62 & 12.74 & 20.54 & 33.72 \\
\hline $\begin{array}{l}\text { F. alcalina } \\
\text { (U/L) }\end{array}$ & 319.15 & 59.36 & 230.59 & 481.82 & 328.64 & 265.39 & 276.65 & 312.86 \\
\hline $\begin{array}{l}\text { Gama-GT } \\
\text { (U/L) }\end{array}$ & 95.65 & 54.86 & 81.62 & 150.24 & 102.64 & 172.82 & 78.56 & 80.83 \\
\hline
\end{tabular}

nejo y alimentación (heno de alfalfa y agua ad libitum) en las instalaciones del Laboratorio de Biotecnología Reproductiva, FMV.

El semen fue colectado a los cuatro machos semanalmente y por cuatro semanas. Para esto, el semen fue extraído por electro-eyaculación siguiendo los protocolos descritos por Director et al. (2007), donde los animales fueron sedados con $0.2 \mathrm{mg} / \mathrm{kg}$ de xilacina y $1.5 \mathrm{mg} / \mathrm{kg}$ de ketamina por vía endovenosa. Se utilizó un electroeyaculador digital modelo Electrojac (EEUU), obteniéndose entre 3 y $5 \mathrm{ml}$ de semen por animal.
Las muestras de semen fresco fueron centrifugadas a $3000 \mathrm{~g} \mathrm{x} 30$ minutos para la separación del plasma seminal. E1 sobrenadante obtenido (1-2 ml) fue evaluado para determinar la ausencia de espermatozoides. La mitad de cada muestra de plasma seminal fue procesada de inmediato y la otra mitad fue guardada en congelación en nitrógeno líquido $\left(-196^{\circ} \mathrm{C}\right)$. Estas muestras fueron descongeladas después de un mes de almacenamiento para su análisis bioquímico.

Se determinaron los niveles de glucosa, colesterol total, colesterol-HDL, triglicéridos, 
Cuadro 2. Comparación bioquímica del plasma seminal fresco y descongelado de cuatro alpacas

\begin{tabular}{lcccc}
\hline & \multicolumn{2}{c}{ Plasma seminal fresco } & \multicolumn{2}{c}{$\begin{array}{c}\text { Plasma seminal } \\
\text { descongelado }\end{array}$} \\
\cline { 2 - 5 } & Promedio & D.E. & Promedio & D.E. \\
\hline Glucosa (mg/dL) & 8.22 & 0.77 & 8.53 & 1.22 \\
Colesterol (mg/dL) & 79.79 & 5.64 & 81.74 & 3.79 \\
Triglicéridos (mg/dL) & $44.12^{\mathrm{a}}$ & 7.38 & $27.31^{\mathrm{b}}$ & 4.65 \\
HDL colesterol (mg/dL) & 4.73 & 0.30 & 4.96 & 0.36 \\
Proteína total (g/dL) & 2.36 & 0.15 & 2.29 & 0.23 \\
Albúmina (g/dL) & 0.97 & 0.33 & 0.81 & 0.06 \\
Calcio (mg/dL) & 11.77 & 1.74 & 11.44 & 0.55 \\
ALT (U/L) & 17.92 & 9.09 & 17.33 & 16.57 \\
F. alcalina (U/L) & 288.76 & 279.59 & 279.86 & 200.76 \\
Gama-GT (U/L) & 89.62 & 39.09 & 114.69 & 38.38 \\
\hline a,b Superíndices diferentes dentro de filasindican diferencia estadística $(\mathrm{p} \varangle 0.05)$ &
\end{tabular}

proteínas totales, albúmina, calcio, alanino aminotrasnferasa, fosfatasa alcalina y gamma glutamil transpeptidasa, a través de kits comerciales específicos para cada determinación (FAR Diagnostics, Italia), siguiendo las especificaciones del fabricante, y por medio de un analizador bioquímico semiautomático (SINOWA, China).

En el caso de las muestras almacenadas, una vez descongeladas se procedió a determinar los parámetros antes mencionados mediante análisis cinético $\mathrm{y}$ fotocolirimétrico, evaluándose las posibles variaciones que hayan sufrido producto de la congelación.

Los resultados se expresan como medias y desviaciones estándares. Las diferencias estadísticas entre los valores de los niveles bioquímicos de plasma seminal fresco y descongelado fueron determinadas con la prueba de T-Student pareado con un nivel de significancia de $\mathrm{p}<0.05$.

\section{Resultados}

En el Cuadro 1 se muestran los valores bioquímicos del plasma seminal de las cuatro repeticiones para cada una de las cuatro alpacas. Solo se encontró una disminución significativa $(\mathrm{p}<0.05)$ de los niveles de triglicéridos $(\mathrm{mg} / \mathrm{dL})$ entre el plasma seminal fresco y descongelado para los animales 1 y 2.

En el Cuadro 2 se presentan los promedios de los valores bioquímicos del plasma seminal de los cuatro animales. En forma similar, solo se encontró una disminución significativa $(\mathrm{p}<0.05)$ de los niveles de triglicéridos $(\mathrm{mg} / \mathrm{dL})$ entre el plasma seminal fresco y descongelado.

\section{Discusión}

Los diversos componentes bioquímicos del plasma seminal no evidenciaron diferen- 
cias significativas por efecto de la congelación y descongelación en comparación con los niveles en fresco. El único componente que mostró modificaciones en su concentración fueron los triglicéridos, los cuales disminuyeron significativamente $(\mathrm{p}<0.05)$ de 44.12 en plasma seminal fresco a 27.31 en plasma seminal descongelado. Este resultado sugiere una cierta labilidad de este componente por el efecto de la congelación y descongelación, tal y como fue demostrado por Paltiel et al. (2008) en plasma seminal humano.

Es posible que los triglicéridos sean más lábiles en un medio como el plasma seminal, pero se sabe que son bastante estables en el plasma sanguíneo, pues no hubo cambios importantes al haberse sometido a cambios de temperatura por 7 días a $4{ }^{\circ} \mathrm{C}$ y hasta 3 meses a $-20^{\circ} \mathrm{C}$ (Henry et al., 1991; Burtis yAshwood, 1994; Tietz, 1994).

La función de los triglicéridos está relacionada a la energía que proporcionan a los espermatozoides durante el proceso de inmovilidad durante su almacenamiento y durante la fase de regeneración después de recuperar la motilidad (Lahnsteiner et al., 1993). Otros estudios han demostrado que los bajos niveles de triglicéridos durante la fase de almacenamiento ocasionan disminución de los recursos energéticos necesarios para la fecundidad, pues el espermatozoide será incapaz de movilizarse adecuadamente (Tekin et al., 2003; Faramarzi, 2012).

Los niveles normales de triglicéridos no se relacionan con la alta calidad de los parámetros espermáticos. Sin embargo, puede interferir con la relación entre la función espermática y la capacidad de fertilización. Diaz-Fontdevila et al. (1993) hallaron que la hipercolesterolemia y la hipertrigliceridemia estaban relacionadas con la disminución de la capacidad de la reacción del acrosoma del espermatozoide en conejos.

La posibilidad de que los bajos niveles de triglicéridos sean la causa de fallas en la inseminación artificial es una interrogante que queda como aporte del presente estudio. Çevk et al. (2007) reportó que bajos niveles de triglicéridos en el plasma seminal están asociados a alteraciones espermáticas como la oligoastenozoospermia en toros.

\section{Literatura Citada}

1. Burtis C, Ashwood E. 1994. Tietz textbook of clinical chemistry. $2^{\text {nd }}$ ed. Philadelphia: Saunders. 1917 p.

2. Bustinza AV. 2001. La alpaca: crianza, manejo y mejoramiento. Tomo II. Puno: Oficina de Recursos del Aprendizaje, Universidad Nacional del Altiplano. 343 p.

3. Cevk M, Tuncer P, Taşdemir $U$, Özgürtaş T. 2007. Comparison of spermatological characteristics and biochemical seminal plasma parameters of normozoospermic and oligoasthenozoospermic bulls of two breeds. Turk $\mathbf{J}$ Vet Anim Sci 31: 381-387.

4. Cross NL. 1998. Role of cholesterol in sperm capacitation. Biol Reprod 59: 711. doi: $10.1095 /$ biolreprod59.1.7

5. De Alba J. 1985. Reproducción animal. México: Ed Copilco. 437 p.

6. Diaz-Fontdevilla M, Bustos-Obregon E. 1993. Cholestrol and polyunsaturated acid enriched diet: Effects on kinetics of the acrosome reaction in rabbit spermatozoa. Mol Reprod Dev 35: 176180.

7. Director A, Giuliano S, Carretero M, Pinto M, Trasorras V, Miragaya M. 2007. Electroejaculation and seminal characteristics in llama (Lama glama). J Camel Pract Res 14: 203-206.

8. Ergün A, Köse SK, Aydos K, Ata A, Avci A. 2007. Correlation of seminal parameters with serum lipid profile and sex hormones. Arch Androl 53: 21-23. doi: 10.1080/01485010600888961

9. Faramarzi M. 2012. Assessment of reproductive parameters in silver carp (Hypophthalmichthys molitrix). World J Fish Marine Sci 4: 244-248. doi: 10.5829/idosi.wjfms.2012.04.03.61325 
10. Ferré L, Werkmeister A. 1996. Desarrollo de una vagina artificial termoeléctrica para la colecta de semen. Rev Agr Prod Anim 16: 363-365.

11. Garnica J, Achata R. 1998. Constituyentes químicos del plasma seminal de la alpaca. En: XII Reunión APPA. Lima: Asociación Peruana de Producción Animal.

12. Henry JB. 1991. Clinical diagnosis and management by laboratory methods. $18^{\circ}$ ed. Philadelphia: Saunders. 1454 p.

13. Herrera E. 1986. Evaluación de dilutores para la conservación de semen en ovinos. Tesis de Bachiller. Puno: Univ Nacional del Altiplano. 72 p.

14. Lahnsteiner F, Patzner RA, Weismann T. 1993. Energy resources of spermatozoa of the rainbow trout, Oncorhynchus mykiss. (Pisces: Teleostei). Reprod Nutr Dev 33: 349-360. doi: 10.1051/rnd:19930404

15. Loomis PR. 2006. Advanced methods for handling and preparation of stallion semen. Vet Clin North Am Equine Pract 22: 663-676.

16. Muiño-Blanco T, Pérez-Pé R, Cebrián-Pérez JA, 2008. Seminal plasma proteins and sperm resistance to stress. Reprod Domes Anim 43 (Suppl 4): 18-31. doi: 10.1111/j.14390531.2008.01228.x
17. Paltiel L, Rønningen K, Meltzer H, Baker S, Hoppin J. 2008. Evaluation of freeze thaw cycles on stored plasma in the Biobank of the Norwegian mother and child cohort study. Cell Preserv Technol 6): 223-229. doi:10.1089/ cpt.2008.0012.

18. Sumar J. 1997. Avances y perspectivas en reproducción de camélidos. I Symposium Internacional Avances en Reproducción de Rumiantes. Lima. 30 p.

19. Tekin N, Secer S, Akcay E, Bozkurt Y, Kayam S. 2003. The effect of age on spermatological properties in rainbow trout (Oncorhynchus mykiss W. 1792). Turk J Vet Anim Sci 27: 37-44.

20. Tietz NW. 1994. Clinical guide to laboratory tests. $2^{\text {a }}$ ed. Philadelphia: Saunders. $476 \mathrm{p}$.

21. Töpfer-Petersen E, EkhlasiHundrieser M, Tsolova M, Leeb T, Kirchhoff C, Müller P. 2005. Structure and function of secretory proteins of the male genital tract. Andrologia 37: 202204. doi: 10.1111/j.1439-0272.2005. 00688.x

22. Troedsson MH, Desvousges A, Alghamdi AS, Dahms B, Dow CA, Hayna J, Valesco R, et al. 2005. Components in seminal plasma regulating sperm transport and elimination. Ani Reprod Sci 89: 171-186. 